Chirurg 2013 $\cdot 84: 62$

DOI 10.1007/s00104-012-2438-z

Online publiziert: 16. Dezember 2012

(c) Springer-Verlag Berlin Heidelberg 2012

J. Reibetanz · C.-T. Germer

Klinik für Allgemein-, Viszeral-, Gefäß- und Kinderchirurgie, Universitätsklinikum Würzburg

\title{
Kolorektales Karzinom im Stadium IV unter palliativer Chemotherapie
}

\section{Günstiger Einfluss der Primärtumorresektion auf das Überleben}

\section{Ergebnisse}

Von den 216 eingeschlossenen Patienten war bei 156 Patienten (72\%) eine Primärtumorresektion erfolgt, bei den übrigen 60 Patienten (28\%) verblieb der Primarius in situ. Nach einem medianen Follow-up von 33 Monaten (4-56) war das mediane OS („overall survival“, 16,3 [13,7$19,2]$ vs. 9,5 [7,4-12,5] Monate, $\mathrm{p}<0,0001)$, die 2-Jahres-OS-Rate (24\% [17-32] vs. $10 \%$ [5-21], $\mathrm{p}<0,0001)$, das mediane PFS („progression-free survival“, 5,1 [4,6-5,6] vs. $2,9[2,2-4,1]$ Monate, $\mathrm{p}<0,001)$ und die 6-Monate-PFS-Rate (38\% [31-46] vs. $22 \%$ [13-34], $\mathrm{p}<0,001)$ jeweils signifikant verlängert/erhöht zugunsten der resezierten Patienten. In der multivariaten Analyse bestätigte sich die Resektion des Primärtumors als der stärkste unabhängige Prognosefaktor für ein verlängertes OS (Risikoreduktion 58\%). Diese Ergebnisse änderten sich nicht, wenn die jeweilige Analyse unter Ausschluss der Patienten mit Rektumkarzinom $(n=43)$ erfolgte.

\section{Methode}

Die FFCD-9601-Studie ist eine multizentrische, randomisierte Phase-III-Studie zum Vergleich verschiedener Protokolle in der First-line-Mono-Chemotherapie beim irresektabel metastasierten CRC. Von den insgesamt 294 in die FFCD-9601Studie eingeschlossenen Patienten präsentierten sich 216 (73\%) mit einer synchronen Metastasierung, diese Patienten bildeten das Studienkollektiv der hier vorliegenden Arbeit.

\section{Diskussion und Fazit}

Die vorliegende Studie belegt, dass die Primärtumorresektion im (irresektabel metastasierten) Stadium IV des CRC unter Erstlinienchemotherapie einen günstigen Einfluss auf das Überleben haben kann. Als Post-hoc-Analyse der FFCD-9601-Studie zur (palliativen) Chemotherapie wurden in der aktuellen Analyse jedoch nur die Patienten be- rücksichtigt, die diese Therapie auch tatsächlich erhielten. Das Outcome derjenigen Patienten, die postoperativ einen ungünstigen Verlauf nahmen und aufgrund von Komplikationen die palliative Chemotherapie erst verspätet (oder gar nicht mehr) erhielten, bleibt unklar. Die chirurgische Erfahrung lehrt, dass dieses Szenario auf einen nicht unerheblichen Prozentsatz unserer Patienten zutrifft. In diesem Zusammenhang sei auch auf die aktuell rekrutierende SYNCHRONOUSStudie [1] verwiesen, eine Multicenterstudie zum Einfluss der Primärresektion vor Beginn einer Chemotherapie auf den Krankheitsverlauf beim synchron metastasierten Kolonkarzinom in der Palliativsituation.

\section{Korrespondenzadresse}

Prof. Dr. C.-T. Germer

Klinik für Allgemein-, Viszeral-, Gefäß- und Kinderchirurgie, Universitätsklinikum Würzburg, Oberdürrbacher Str. 6, 97080 Würzburg germer_c@klinik.uni-wuerzburg.de

Interessenkonflikt. Der korrespondierende Autor gibt für sich und seinen Koautor an, dass kein Interessenkonflikt besteht.

\section{Literatur}

1. Rahbari NN et al (2012) Resection of the primary tumour versus no resection prior to systemic therapy in patients with colon cancer and synchronous unresectable metastases (UICC stage IV): SYNCHRONOUS - a randomised controlled multicentre trial (ISRCTN30964555). BMC Cancer 12:142 